University of Windsor

Scholarship at UWindsor

1996

\title{
Role of Bicaudal-D in patterning the Drosophila egg chamber in mid-oogenesis
}

\author{
Andrew Swan \\ University of Windsor
}

B. Suter

Follow this and additional works at: https://scholar.uwindsor.ca/biologypub

Part of the Biology Commons

\section{Recommended Citation}

Swan, Andrew and Suter, B., "Role of Bicaudal-D in patterning the Drosophila egg chamber in midoogenesis" (1996). Development, 122, 11, 3577-3586.

https://scholar.uwindsor.ca/biologypub/1120

This Article is brought to you for free and open access by the Department of Biological Sciences at Scholarship at UWindsor. It has been accepted for inclusion in Biological Sciences Publications by an authorized administrator of Scholarship at UWindsor. For more information, please contact scholarship@uwindsor.ca. 


\title{
Role of Bicaudal-D in patterning the Drosophila egg chamber in mid- oogenesis
}

\author{
Andrew Swan and Beat Suter \\ Department of Biology, McGill University, 1205 Dr Penfield Avenue, Montreal, PQ, Canada, H3A 1B1
}

\section{SUMMARY}

The Bicaudal-D (Bic-D) gene is required early in Drosophila oogenesis for the differentiation of an oocyte from one of a cluster of $\mathbf{1 6}$ interconnected germarial cells. To analyze the role of Bic-D later in oogenesis, we have constructed Drosophila lines in which Bic-D expression is under the control of the $h s p 70$ promoter. In these flies, Bic$D$ activity can be induced early in oogenesis, allowing an oocyte to be made. Then, by shifting females to noninducing conditions, Bic-D levels are depleted for the remainder of oogenesis. Using this system, we find that Bic$D$ is indeed required in the later stages of oogenesis. In ovaries from mutant females, oocyte growth is reduced, apparently due to defects in nurse-cell-to-oocyte transport. Smaller oocyte size results in the misalignment of follicle cells and the underlying germ line, leading to ventralization of dorsal follicle cells and to defects in centripetal cell migration. In addition, we show that $B i c-D$ is required for the localization of specific mRNAs at both the anterior and posterior of the oocyte.

Key words: Drosophila, oogenesis, cytoskeleton, axis formation, RNA localization, Bicaudal-D (Bic-D), egg chamber, follicle cell, nurse cell

\section{INTRODUCTION}

Asymmetric localization of patterning factors within cells often underlies developmental patterning, and Drosophila oogenesis has proven to be a valuable system for studying how such asymmetries arise. The Drosophila ovary has been divided into 3 germarial regions and 13 vitellarial stages. In the germarium, a cystoblast undergoes four incomplete divisions to produce a 16-cell cyst in which all cells are connected by cytoplasmic bridges. Beginning in region $2 \mathrm{~b}$, a microtubule organizing center (MTOC) appears in one of these cells and extends microtubules through the ring canals into the other 15 cells. Specific mRNAs and proteins accumulate in this cell and it differentiates as an oocyte. The other 15 cells become nurse cells and function in providing material for the growing oocyte (reviewed in Cooley and Theurkauf, 1994). Microtubule-destabilizing drugs disrupt the accumulation of factors in the pro-oocyte and result in the failure to differentiate an oocyte (Koch and Spitzer, 1983; Theurkauf et al., 1993). This evidence supports a model in which a polarized microtubule network directs the transport of specific factors into the future oocyte. Among these would be factors that promote oocyte differentiation.

During stages 7 and 8, the oocyte MTOC disappears and microtubules reorganize within the oocyte such that minus ends are found mainly at the anterior and plus ends extend towards the posterior (Theurkauf et al., 1992). This polarized microtubule network is thought to direct the anterior and posterior transport of specific factors within the oocyte (see Cooley and Theurkauf, 1994). Transcripts encoding the anterior morphogen, bicoid, accumulate at the anterior of the oocyte in stage 8 and remain at this site throughout oogenesis. Several other transcripts, including Bic-D, orb and $f_{s}(1) K 10$ mRNAs also accumulate at the anterior of the oocyte at this time and remain at this location until late in stage 10. Also in stage 8, staufen protein and oskar (osk) mRNA migrate together to the posterior pole of the oocyte where osk is then translated and functions to recruit other posterior factors. Accumulation of these anteriorly and posteriorly localized transcripts is disrupted by treating egg chambers with microtubule-destabilizing drugs (Pokrywka and Stephenson, 1991, 1995; Clark et al., 1994), suggesting that microtubule-based transport and/or anchoring is involved in their localization. This model is supported by the observation that in females expressing a transgenic kinesin- $\beta$-gal fusion protein, the fusion protein accumulates transiently at the posterior pole of the oocyte at the same time that osk mRNA and staufen protein are first detected there (Clark et al., 1994).

During the stages when A/P patterning is set up, the first signs of D/V polarity in the egg chamber are detected. In stage 8, grk mRNA associates with the oocyte nucleus and moves with it to an anterior/cortical position within the oocyte. Localized grk protein is thought to signal overlying follicle cells through the top receptor, initiating a signal transduction cascade involving members of the Ras/Raf/MAP Kinase pathway (reviewed in Schüpbach and Roth, 1994). Late in oogenesis, the follicle cells that surround the maturing egg produce the vitelline membrane and chorion. Dependent on grk/top signaling, the dorsal anterior follicle cells produce specialized dorsal chorion structures, the dorsal appendages and the operculum.

The product of the Bicaudal-D (Bic-D) gene may play a 
role in a number of patterning processes during oogenesis. In ovaries from females homozygous or hemizygous for loss-offunction alleles of Bic-D, no oocyte is made and all 16 cells of the germarial cyst adopt a nurse cell fate (Mohler and Wieschaus, 1986). The MTOC, which normally forms in the oocyte, is not detected in these mutants and oocyte-specific mRNAs fail to accumulate in a single cell (Suter and Steward, 1991; Ran et al., 1994; Theurkauf et al., 1993). These observations may point to a role for Bic-D in microtubule-based transport or anchoring early in oogenesis.

The phenotypes produced by two dominant alleles of Bic$D$ suggest that this gene also functions later in oogenesis, in both $\mathrm{A} / \mathrm{P}$ and $\mathrm{D} / \mathrm{V}$ patterning of the egg chamber. Females carrying either Bic-D $D^{71.34}$ or Bic-D $D^{I I E 48}$ produce embryos missing anterior structures and instead possessing a mirrorimage duplication of posterior structures (Mohler and Wieschaus, 1986). This defect appears to result from a mislocalization of osk mRNA to the anterior pole of the oocyte late in oogenesis (Ephrussi et al., 1991; Kim-Ha et al., 1991). This result, along with the finding that the earlier localization of osk mRNA to the pro-oocyte is blocked in Bic-D $D^{\text {null }}$ mutants (Ran et al., 1994), may indicate a role for Bic-D in osk mRNA localization later in oogenesis. The two dominant Bic- $D$ alleles also display a recessive fused dorsal appendage phenotype similar to that produced by weak alleles of $g r k$ and top (Mohler and Wieschaus, 1986). Therefore, there is evidence that Bic-D is involved in the establishment of both primary axes.

Bic-D $D^{71.34}$ and Bic-D $D^{I I I E 48}$ are dominant gain-of-function mutations and thus their phenotypes may not be indicative of actual Bic-D function. To understand the role of Bic-D in patterning of the egg chamber, it is essential to determine the loss-of-function phenotypes for Bic-D during these stages. So far this has not been possible because all recessive alleles of Bic-D prevent the formation of an oocyte. Study of the later requirements for $B i c-D$ would be possible if Bic-D activity could be provided early in oogenesis, permitting an oocyte to be made, but then removed for the remainder of oogenesis. We have accomplished this by introducing an inducible Bic-D transgene into female Drosophila otherwise lacking Bic-D activity. Using this method, we find that Bic$D$ is required for oocyte growth and both $\mathrm{D} / \mathrm{V}$ and $\mathrm{A} / \mathrm{P}$ patterning of the egg chamber. Our results also provide evidence for a prepatterning of follicle cell fates independent of the underlying oocyte.

\section{MATERIALS AND METHODS}

\section{Construction of Bic-D mid-oogenesis mutants (Bic-Dmom)}

The $3.6 \mathrm{~kb}$ ClaI/EcoRI fragment from Bic-D cDNA c18 (Suter et al., 1989), which includes the entire open reading frame, was cloned as a leader fusion behind the $h s p 70$ promoter in the pElba vector (a gift from Paul Schedl, Princeton). This vector possesses scs and scs' boundary domains to minimize chromosomal position effects (Kellum and Schedl, 1991). The fusion gene was then inserted into a $\mathrm{pCaSpeR}$ vector and introduced into flies by P-element-mediated transformation. The results described here are from experiments performed on one line, $P\left[w^{+} h s B i c-D\right]-94$, though the same phenotypes were seen with two other independently derived lines. To generate Bic-D mid-oogenesis mutant $\left(B i c-D^{\text {mom }}\right)$ females, progeny from the cross, $w ; D f(2 L) T W 119 / C y O ; P\left[w^{+} h s B i c-D\right]-94 /+\times w ; B i c-$
$D^{r 8} / \mathrm{CyO}$ were given two $37^{\circ} \mathrm{C}$ heat shocks of 30 minutes per day starting late in the 3rd larval instar or at the early pupal stage, and lasting for 3 to 4 days. The lethality associated with Bic-D $D^{\text {null }}$ mutations (Ran et al., 1994) is rescued by this induction of the transgene. These flies were then transferred to $18^{\circ} \mathrm{C}$ and $\mathrm{Bic}$ $D^{r 8} / D f(2 L) T W 119 ; \quad P\left[w^{+} h s B i c-D\right]-94 /+\left(B i c-D^{m o m}\right)$ females were selected and examined for oogenesis and egglay phenotypes at various times after the shift. Sibling females of the genotype, $w ; B i c$ $D^{r 8}$ (or $\left.D f(2 L) T W 119\right) / C y O ; P\left[w^{+} h s B i c-D\right]-94 /+$ were processed in parallel and used as controls in all experiments except in the protein blot analysis and immunostaining with anti-Bic-D antibodies. For these experiments, OregonR flies were used as controls. For all experiments involving examination of ovary phenotypes, females were aged 2 to 4 days on standard food with live yeast prior to ovary dissection.

\section{Protein blot analysis}

Protein blots were performed as described in Suter and Steward (1991) using ovary extracts pooled from five females for each sample. All Bic-D protein detected in these ovaries is produced by the transgene, since the $B i c-D^{r 8}$ allele makes very low levels of a truncated protein (Ran et al., 1994).

\section{Immunostainings}

Ovaries from Bic- $D^{m o m}$ and OregonR females, collected at various times after shift to $18^{\circ} \mathrm{C}$, were fixed and immunostained as previously described (Suter and Steward, 1991). To control for variability in fixation or staining, mutant and control ovaries were processed together in the same tube. Either control or mutant ovary pairs were separated into two to allow them to be distinguished later. Pictures were taken from single optical sections using a Leica confocal laser scanning microscope.

\section{Ovary RNA in situ hybridizations}

RNA in situ hybridizations were performed as described previously (Suter and Steward, 1991). Control and mutant ovaries were processed together.

\section{Determination of chorion phenotypes}

Eggs were collected from overnight egg lays and classified according to the severity of their chorion phenotype as described in Fig. 2. For photographing eggs with representative chorion phenotypes, eggs were mounted in Hoyers medium and photographed under dark field as described in Wieschaus and Nüsslein-Volhard (1986).

\section{Enhancer trap detection}

The BB127 and BB142 enhancer trap lines are described in Schüpbach and Roth (1994). The BB142 enhancer trap insertion on $2 \mathrm{~L}$ was recombined onto a $B i c-D^{r 8}$ chromosome and crossed to $D f(2 L) T W 119 / C y O ; \quad P\left[w^{+} h s B i c-D\right]-94 /+$. The 3rd chromosome BB127 insert was crossed into a Bic- $D^{\text {mom }}$ background. $\beta$-gal detection was performed as in Lis et al. (1983).

\section{Egg chamber size analysis}

Control and Bic- $D^{m o m}$ egg chambers were fixed in $4 \%$ paraformaldehyde in PBS $+0.2 \%$ Tween and stained with rhodamine phalloidin (Molecular Probes). Phalloidin labels actin at cell membranes and, therefore, allows us to easily trace cells. Individual egg chambers were scanned using a Leica confocal laser scanning microscope. Single images from optical sections giving the largest oocyte areas were measured using NIH imaging software. For each egg chamber, the ratio of oocyte area to total area (oocyte + nurse cells) was determined and, for all of the eggs of a given stage, these values were averaged. Egg chambers were staged according to follicle cell morphology. 


\section{RESULTS}

An inducible $B i c-D$ transgene allows examination of $B i c-D$ requirements in late oogenesis

To examine the Bic-D loss-of-function phenotype in later oogenesis, we have placed the Bic-D coding sequence under the control of the $h s p 70$ promoter and introduced this transgene into females otherwise lacking Bic-D activity. $s c s$ and $s c s^{\prime}$ boundary domains (Kellum and Schedl, 1991) flanking the transgene help ensure strict heat-shock control. When Bic-D is induced in these Bic-D $D^{\text {null }}$; $h$ s-Bic-D females by providing them with regular heat shocks, they are fertile and lay mainly wildtype eggs. Total Bic-D protein levels in ovaries under these conditions are approximately half of wild-type levels (Fig. 1A). Immunostaining of these Bic-D $D^{\text {null }} ;$ hs-Bic-D ovaries with antiBic-D antibodies reveals that early in oogenesis, Bic-D protein is localized to the oocyte at levels similar to those seen in wild type (Fig. 1B). Stage 8 and later egg chambers, however, contain very low levels of Bic-D protein in the germ line (data not shown), possibly indicating that the $h s p 70$ promoter is not as strongly inducible in the later stages of oogenesis. In addition to expression of Bic-D in the germ line, induction of the transgene also directs the expression of Bic-D in the somatically derived follicle cells which surround the 16-cell cyst. This somatic expression of Bic-D appears to have no adverse effect on oogenesis since no mutant phenotypes are observed upon induction of the transgene in control females carrying one copy of the endogenous Bic-D gene (data not shown).

Within one and a half days after shifting flies to noninducing conditions $\left(18^{\circ} \mathrm{C}\right)$, Bic-D protein levels fall noticeably and, by 5.5 days, Bic-D is almost undetectable by protein blotting or immunostaining (Fig. 1A,B). Correlating with a reduction in Bic-D protein levels after shift to $18^{\circ} \mathrm{C}$, egg chambers start to display a 16-nurse-cell phenotype like that observed in Bic-D recessive mutants. After 12 to 13 days under non-inducing conditions, ovaries from these Bic- $D^{\text {null }} ; h$ s-Bic$D$ females mainly consist of 16-nurse-cell chambers. Sometimes a few mature eggs are also present, representing the last egg chambers in which Bic-D levels were sufficient to make an oocyte.

Taken together, these results show that the $h s-B i c-D$ transgene provides an inducible source of Bic-D activity, which can rescue the block at oocyte determination in Bic- $D^{\text {null }}$ females. Several days after shift to $18^{\circ} \mathrm{C}$, we can examine egg chambers in which Bic-D activity was initially sufficient to permit oocyte determination, but was reduced or absent for the remainder of oogenesis. This allows us to determine the requirements for $B i c-D$ after its role in oocyte determination. In the remainder of this paper, we will refer to Bic-D $D^{\text {null }} ; h_{s-}$ Bic-D females shifted down to $18^{\circ} \mathrm{C}$ as Bic-D mid-oogenesis mutants or Bic-Dmom and the oogenesis phenotypes that they display as Bic- $D^{m o m}$ phenotypes.

\section{Chorion defects in Bic- $D^{\text {mom }}$ egg chambers}

$B i c-D^{\text {mom }}$ females lay eggs with phenotypes ranging from partially fused or fused dorsal appendages to reduced or absent dorsal appendages (Fig. 2A-C). In many cases, the dorsal appendage remnants are located more posteriorly on the eggshell than in wild type. In addition to dorsal appendage defects, another dorsal chorion structure, the operculum, is
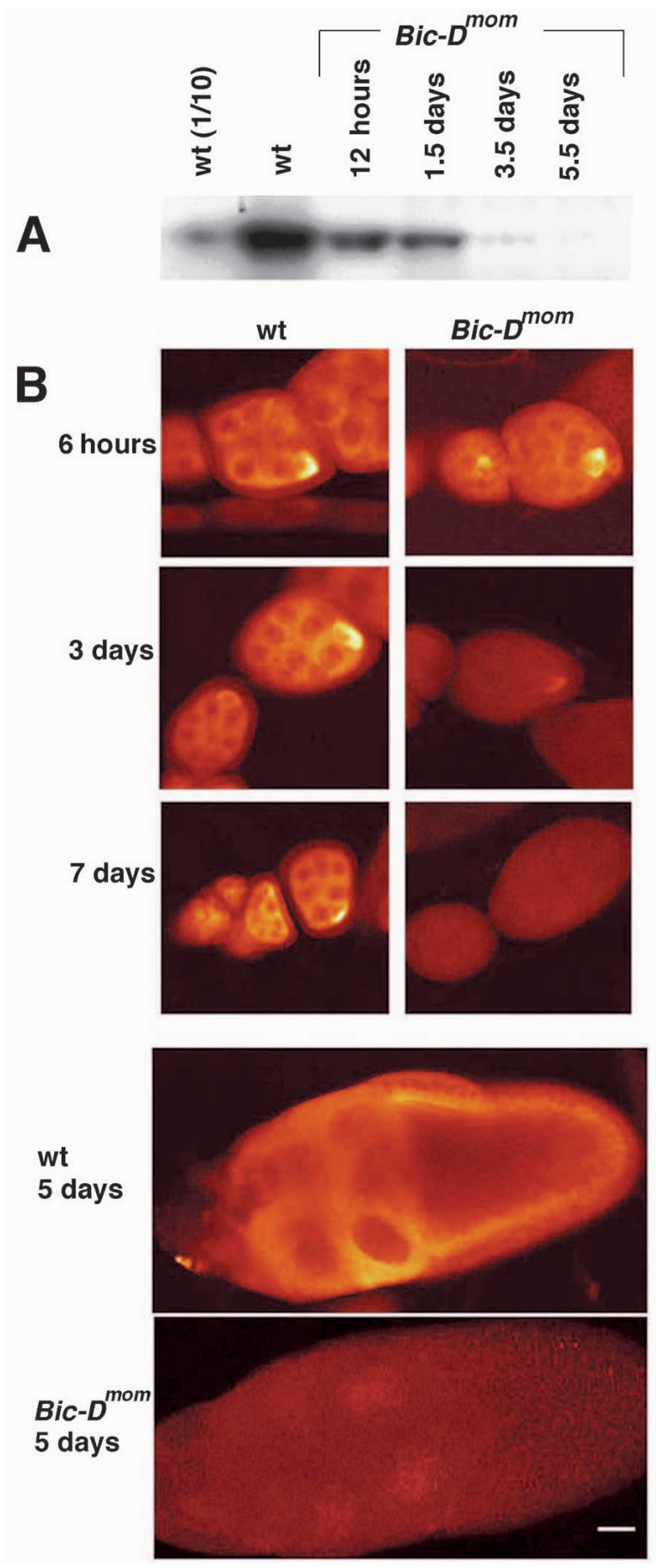

Fig. 1. Induction of Bic-D protein in Bic- $D^{\text {null }} ; h s$-Bic-D (Bic- $\left.D^{\text {mom }}\right)$ ovaries. (A) Protein blot of ovary extracts from wild-type and Bic$D^{\text {mom }}$ females at various times after last induction of Bic-D. With the exception of lane 1 , which contains $1 / 10$ the amount loaded in lane 2, equal amounts of protein were loaded in each lane as determined by Ponceau S staining (not shown). 12 hours after the last heat shock, protein levels are approximately half of wild-type levels. By $5 \frac{1}{2}$ days, Bic-D protein is almost undetectable.

(B) Confocal laser scanning images of wild-type and Bic-D ${ }^{\text {mom }}$ egg chambers at different times after shift to $18^{\circ} \mathrm{C}$. Under heat-shock conditions, the amount and distribution of Bic-D protein in early stage egg chambers is similar to that in wild type. Bic-D levels fall sharply following shift to $18^{\circ} \mathrm{C}$, and only background signal can be detected by 5 days. Scale bar, $10 \mu \mathrm{m}$. 

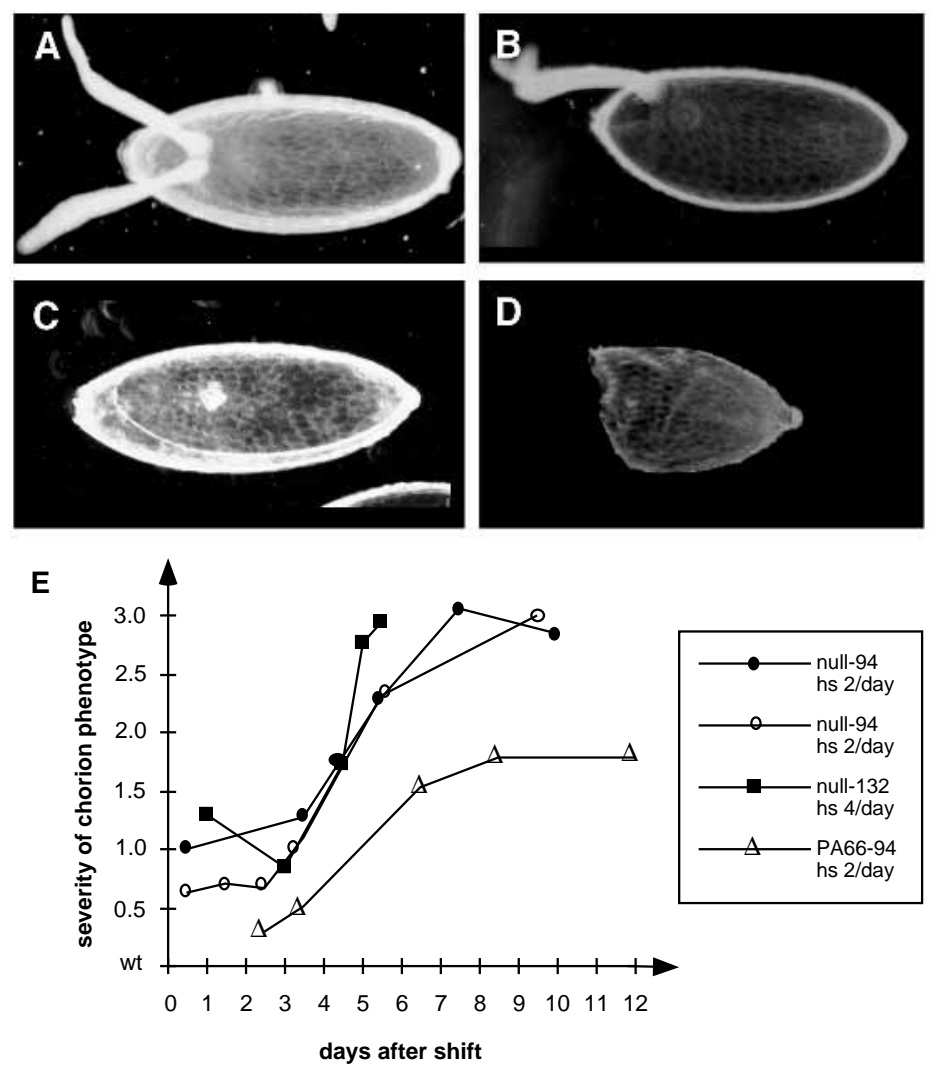

often reduced or missing, and the follicle cell imprints on the dorsal surface of the egg are rounded, resembling those normally seen on the ventral surface. Mutant females also lay eggs that are small and often lack anterior chorion (Fig. 2D). All of these mutant phenotypes can be ordered in severity based on when they first appear following the shift to $18^{\circ} \mathrm{C}$ (Fig. 2E).

Control results demonstrate that the chorion phenotypes that we observe in $B i c-D^{m o m}$ eggs are not due to trivial causes such as overexpression of Bic-D, mutations caused by insertion of the transgene or secondary effects resulting from heat shock. First, females heterozygous for Bic-D, with or without the transgene, and subjected to the same heat-shock regimen produce wild-type-looking eggs (data not shown). Second, all phenotypes are rescued by inducing Bic-D expression from the transgene (Fig. 2A,E). Third, the same phenotypes are observed in three independent transgenic lines (Fig. 2E and data not shown). Therefore, the dorsal appendage defects that we observe represent a Bic-D loss-of-function phenotype. The two hypomorphic alleles, Bic- $D^{P A 66}$ and Bic$D^{R 26}$, also display a fused dorsal appendage phenotype following rescue of the oocyte determination block. This phenotype is weaker than that observed in a Bic- $D^{\text {null }}$ background (Fig. 2E), indicating that these alleles are also hypomorphs for the chorion phenotype.

Many of the chorion defects in eggs from Bic- $D^{m o m}$ females resemble those observed in grk and top mutants (Schüpbach, 1987), and may indicate a role for Bic-D in the grk/topmediated pathway that determines dorsal follicle cell fates. To determine if Bic-D is involved in the same pathway as $g r k$ in establishing D/V polarity, we first tested for genetic interactions between these two loci. Almost no fused dorsal
Fig. 2. Ventralized chorion phenotypes produced by Bic- $D^{\text {mom }}$ females. (A) Wild-type-looking eggshell produced by a Bic- $D^{\text {mom }} 3$ days after last induction of Bic-D. (B-D) Chorion phenotypes from $B i c-D^{m o m}$ eggs 10 days after last induction of Bic-D (B) Fused dorsal appendages. (C) Reduced and posteriorly displaced dorsal appendage material. (D) Open chorion. (E) Severity of chorion phenotypes as a function of time after the last induction of Bic-D. Eggs collected at various timepoints were classified and assigned a value according to the severity of their chorion phenotype: wild type $=0$, partially fused dorsal appendages $=1$, fused dorsal appendages $=2$, reduced or absent dorsal appendages $=3$, open chorion phenotype $=4$. The results for Bic- $D^{\text {null }} ; h s-B i c-D-94$, expressed as percentages (open circles on the graph) are as follows:

\begin{tabular}{lrrrrr}
\hline \multirow{2}{*}{$\begin{array}{l}\text { Days after } \\
\text { heat shock }\end{array}$} & \multicolumn{5}{c}{ \% of eggs in each phenotypic class } \\
\cline { 2 - 5 } $0-1$ & 0 & 1 & 2 & 3 & 4 \\
\hline $1-2$ & 39 & 59 & 2 & 0 & 0 \\
$2-3$ & 36 & 59 & 4 & 2 & 0 \\
$3-4$ & 24 & 58 & 3 & 1 & 0 \\
$5-6$ & 3 & 27 & 13 & 8 & 2 \\
$9-10$ & 3 & 19 & 20 & 27 & 19 \\
\end{tabular}

The values on the graph represent the average severity of the chorion phenotype from a single egg collection. An average of 70 eggs were scored from each collection, 31 eggs for the smallest. Females carrying the Bic-D $D^{\text {null }} ; h s-B i c-D-132$ insert required heat shocks 4 times daily to rescue the fused dorsal appendage phenotype. Results with $B i c-D^{R 26}$ are similar to those obtained with Bic- $D^{P A 66}$ (data not shown).

appendages are observed in eggs produced by females heterozygous for either Bic-D ${ }^{r 8}(2 / 54)$ or $\operatorname{grk}^{H K 36}(0 / 76)$. However, most eggs from females transheterozygous for Bic$D^{r 8}$ and $g r k^{H K 36}$ display a fused dorsal appendage phenotype (46/65). A similar but less penetrant fused dorsal appendage phenotype is observed in eggs from females transheterozygous for a Bic-D deficiency and $g r k^{2 B 6}$ (data not shown). This failure to complement may indicate that the two genes are involved in the same pathway.

If $B i c-D$ is involved in the same pathway as grk, it may be required for the correct localization of grk mRNA within the oocyte. Mutations in the genes orb, $f s(1) K 10$, cappuccino, spire and squid all appear to disrupt $\mathrm{D} / \mathrm{V}$ patterning by causing a mislocalization of grk mRNA (Neuman-Silberberg and Schüpbach, 1993; Roth and Schüpbach, 1994). To determine if $B i c-D$ mutants similarly disrupt $\mathrm{D} / \mathrm{V}$ patterning, we performed RNA in situ hybridizations to grk transcripts in Bic$D^{m o m}$ egg chambers. In these mutants, as in wild type, grk mRNA associates strictly with the oocyte nucleus (Fig. 3A,B), indicating that $B i c-D$ is not required for $g r k$ localization to the periphery of the oocyte nucleus.

While grk mRNA localization is not noticably affected, we observe other defects in these egg chambers that may lead to a disruption in grk/top signaling. By stage 10A in wild-type oogenesis, the oocyte has expanded to occupy approximately half of the egg chamber. The posterior migration of follicle cells, which began in stage 9 , is completed by this stage. As a result, most of the follicle cells form a columnar epithelium over the oocyte, while a small number of highly flattened follicle cells covers the nurse cells. The margin between the oocyte and nurse cells corresponds precisely with the border between flattened and columnar follicle cells. grk message 
Table 1. Oocyte growth is reduced in Bic- $D^{m o m}$ ovaries

\begin{tabular}{|c|c|c|c|c|c|c|}
\hline \multirow[b]{2}{*}{ Stage } & \multicolumn{3}{|c|}{ Control } & \multicolumn{3}{|c|}{ Bic-D $D^{\text {mom }}$} \\
\hline & Total area* & Oocyte area & Ratio** & Total area* & Oocyte area & Ratio** \\
\hline $2-6$ & & & $0.10 \pm 0.02$ & & & $0.06 \pm 0.01$ \\
\hline 7 & 4110 & 671 & $0.16 \pm 0.01$ & 5044 & 316 & $0.06 \pm 0.01$ \\
\hline 8 & 6327 & 1026 & $0.13 \pm 0.03$ & 7435 & 510 & $0.08 \pm 0.01$ \\
\hline 9 & 13546 & 3081 & $0.21 \pm 0.05$ & 13630 & 1959 & $0.12 \pm 0.05$ \\
\hline $10 \mathrm{~A}$ & 26110 & 8901 & $0.34 \pm 0.01$ & 30368 & 9102 & $0.30 \pm 0.02$ \\
\hline $10 \mathrm{~B}$ & 42849 & 15804 & $0.37 \pm 0.03$ & 41888 & 14622 & $0.35 \pm 0.02$ \\
\hline
\end{tabular}

Areas measured in $\mu \mathrm{m}^{2}$

Staging of egg chambers is based on follicle cell morphology. Stages 2-6 are grouped together because they cannot be distinguished based on this criterion. Average areas are not given for these stages because of the large range of egg chamber sizes. However, the ratio of oocyte to total size is constant throughout these stages.

*Area of oocyte + nurse cells.

**The ratio of oocyte area over total area was determined for each egg chamber of a given stage and then the average of these ratios was calculated.

underlies the anteriormost columnar follicle cells, and it is these cells that normally appear to receive grk signal (Schüpbach and Roth, 1994). In stage 10A Bic-D ${ }^{\text {mom }}$ egg chambers, columnar follicle cells still extend over the posterior half of the egg chamber. However, the oocyte is smaller than in wild type and the margin between oocyte and nurse cells is displaced posteriorly relative to the border between flattened and columnar follicle cells (Fig. 3D). As a result, grk signal is displaced relative to the overlying follicle cells. This in turn results in a posterior displacement of follicle cell fates as seen in the expression of the dorsal/anterior follicle cell marker BB142 (Fig. 3F). This indirect effect on grk/top signaling could explain the failure to properly establish dorsal follicle cell fates and demonstrates that the establishment of $\mathrm{D} / \mathrm{V}$ polarity in the egg chamber depends on the proper coordination between oocyte growth and follicle cell migration.

In stage 10 and later Bic- $D^{\text {mom }}$ egg chambers, we often observe a mislocalization of the oocyte nucleus (Fig. 4A). Video analysis of mutant egg chambers reveals that the oocyte nucleus often moves within the oocyte, usually confined to the cortex, during the rapid ooplasmic streaming that begins in stage 10B (data not shown). Some grk mRNA remains associated with the nucleus even when it is mispositioned (data not shown). This subsequent displacement of grk mRNA late in oogenesis may also affect follicle cell fate since BB142 expression, in addition to being displaced posteriorly, is frequently found at low levels in a broad range of follicle cells in stage 10B mutant egg chambers (Fig. 3F).

Dependent on grk/top signaling, dorsal follicle cells are thought to produce a signal required later for the restriction of ventral fates in the embryo, and therefore many mutants that disrupt the specification of dorsal follicle cell identity also disrupt the polarity of the embryo (reviewed in Schüpbach and Roth, 1994). However, no embryonic D/V patterning defects are observed in eggs from Bic- $D^{m o m}$ females. This could simply be due to the fact that most eggs laid by these mutants are not fertilized and those eggs with the most severe chorion defects are never fertilized (data not shown). Alternatively, it is possible that the follicle cells that receive dorsalizing signal in Bic- $D^{\text {mom }}$ egg chambers, while unable to induce proper dorsal appendage formation, are still able to repress ventral fates in the embryo.

In addition to ventralized eggs, Bic- $D^{\text {mom }}$ females produce eggs that are missing anterior chorion (Fig. 2D). Similar open chorion phenotypes have been described for a small number of maternal effect mutants (Schüpbach and Wieschaus, 1991). One of these, Bicaudal-C, also displays a dominant bicaudal phenotype and therefore these two genes share similar recessive and dominant phenotypes. As with the other known open chorion mutants, the Bic- $D^{\text {mom }}$ phenotype appears to be a result of failed centripetal cell migration. Normally in stage $10 \mathrm{~B}$, the anterior columnar follicle cells overlying the oocyte/nurse cell boundary migrate between the oocyte and the nurse cells, separating the maturing egg from the nurse cells. The centripetal cells later contribute towards making the anterior portion of the egg shell. In Bic-D $D^{\text {mom }}$ egg chambers, centripetal cell migration is often incomplete, likely because nurse cells extend into the space where these cells normally would migrate, physically impeding their progress. Consistent with this interpretation, we often see egg chambers in which the nurse cells bordering the oocyte appear to have been displaced far enough posteriorly to allow centripetal cells to intercalate between them and the next set of nurse cells (Fig. 4B,C).

\section{Bic- $D$ requirement in oocyte growth}

The defects in $\mathrm{D} / \mathrm{V}$ patterning and in centripetal cell migration in Bic- $D^{\text {mom }}$ appear to be indirect consequences of a failure in oocyte growth. Throughout most of oogenesis the oocyte nucleus is largely transcriptionally inactive and oocyte growth occurs primarily by uptake of materials from other cells (King and Burnett, 1959). Oocyte growth early in oogenesis appears to occur via a microtubule-dependent process (Koch and Spitzer, 1983), while after stage 7 an actin-dependent and microtubule-independent transport of nurse cell contents into the oocyte has been observed (Bohrman and Biber, 1994). Also beginning in stage 8, the oocyte expands by uptake of yolk from the surrounding follicle cells and hemolymph.

To determine which aspect of oocyte growth is affected in Bic- $D^{\text {mom }}$ egg chambers, we measured oocyte and egg chamber size in mutant and control egg chambers, and for each egg chamber we determined the ratio of oocyte to total egg chamber size (Table 1). This analysis reveals that in Bic- $D^{\text {mom }}$ ovaries, oocyte size is reduced throughout oogenesis, but overall size of the egg chambers is not reduced. Therefore, the reduced oocyte size is not due to an overall growth defect in 

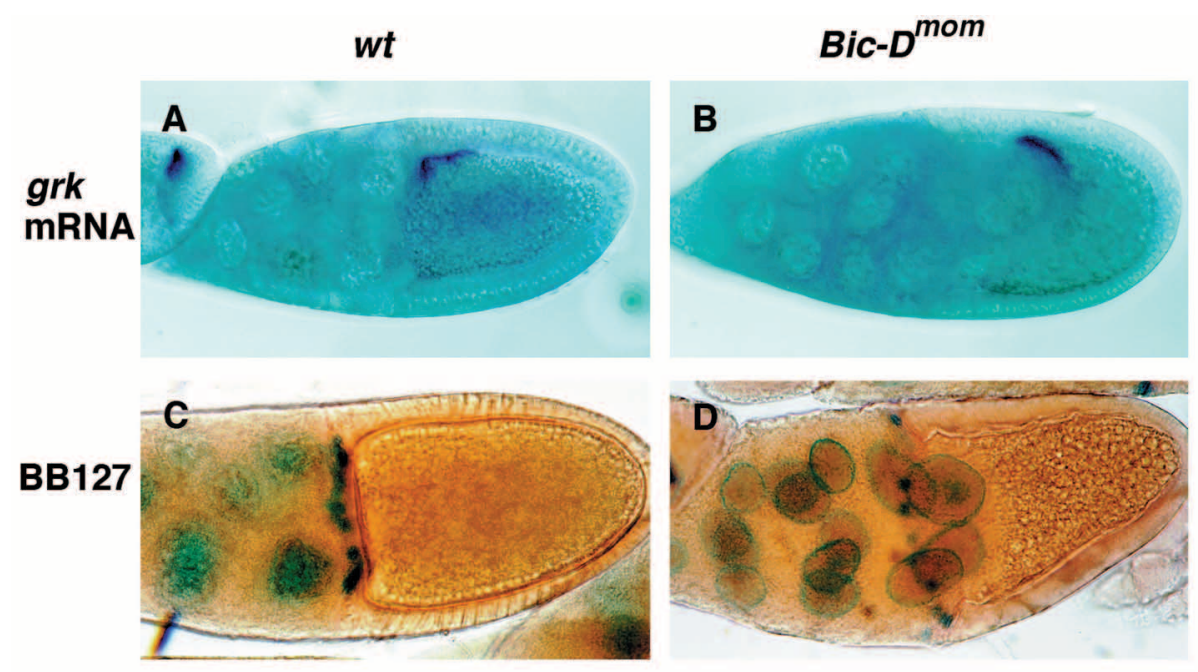

BB142

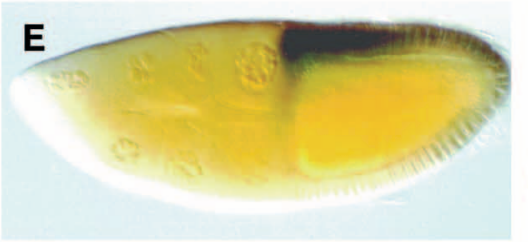

Fig. 3. Ventralized phenotypes in Bic- $D^{\text {mom }}$ egg chambers. (A) In control egg chambers, grk transcript accumulates at a dorsal/anterior position in the oocyte, in proximity to the anterior columnar follicle cells. (B) In Bic-D ${ }^{\text {mom }}$, grk mRNA still associates with the oocyte nucleus at the anterior of the oocyte. In these egg chambers, the oocyte is smaller and its anterior margin is displaced posteriorly. As a result, grk message is no longer located beneath the anteriormost columnar follicle cells. (C) $\beta$-galactosidase expression from the BB127 enhancer trap line in wild-type egg chambers. The marker is expressed in nurse cells and in a row of columnar follicle cells bordering on the anterior flattened follicle cells. This border is precisely at the margin between oocyte and nurse cells. (D) BB127 expression in Bic- $D^{\text {mom }}$ egg chambers. Nurse cells are seen extending past the border between columnar and flattened follicle cells. (E) $\beta$-galactosidase expression from the BB142 enhancer trap line in a control stage 10A egg chamber. Expression is restricted to a set of columnar follicle cells overlying the anterior margin in

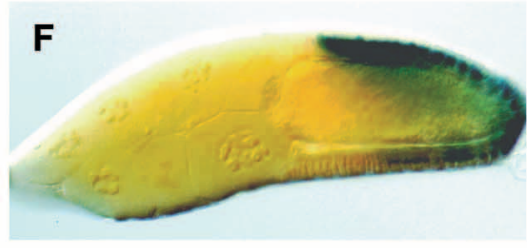
ressing cells is shifted posteriorly. Lower levels the oocyte. (F) BB142 expression in a Bic- $D^{m o m}$ egg chamber. The m
of expression are also seen in a broad range of posterior follicle cells.

the 16-cell germarial cyst or to failure in yolk uptake, but instead is most likely due to reduced transfer of nurse cell contents into the oocyte. The effect on oocyte growth is detected in stages 2 to 6 and is most pronounced in stage 7 . By stage 10B, oocyte size approaches that observed in the heterozygous controls (Table 1). The apparent stage specificity of these defects may reflect a specific requirement for Bic-D in nurse-cell-to-oocyte transport early in oogenesis and, therefore, implicates $B i c-D$ in the putative microtubule-based transport process which may be functioning during these stages.

If $B i c-D$ is required for nurse-cell-to-oocyte transport, the accumulation of oocyte-specific factors may be reduced in $B i c-D^{m o m}$ egg chambers. In wild-type ovaries, orb, $f s(1) K 10$ and $o s k$ mRNAs are localized to the oocyte during the early stages of oogenesis, and this is dependent on microtubules (Theurkauf et al., 1993; Pokrywka and Stephenson, 1995). In Bic-D ${ }^{m o m}$, orb and $f s(1) K 10$ mRNAs still accumulate in the oocyte at normal levels up to stage 7 while osk localization is only partially reduced during these stages (Table 2). We do not know whether the observed early localization of these mRNAs indicates that their localization is Bic-D independent or whether residual Bic-D activity present in these early stages is sufficient for their localization. It is conceivable that the latter may be the case because these egg chambers have sufficient Bic-D activity to make an oocyte and, as shown previously (Ran et al., 1994), residual Bic-D activity can be sufficient to localize certain mRNAs to the presumptive

Table 2. Localization of oocyte specific factors in Bic- $D^{m o m}$ and control egg chambers

\begin{tabular}{|c|c|c|c|c|c|c|c|c|}
\hline \multirow[b]{2}{*}{ Localized factor } & \multirow[b]{2}{*}{ Genotype } & \multicolumn{7}{|c|}{ Oogenesis stage } \\
\hline & & $2-6$ & 7 & 8 & 9 early & 9 late & $10 \mathrm{~A}$ & $10 \mathrm{~B}$ \\
\hline gurken mRNA & $\begin{array}{l}\text { wild-type } \\
\text { Bic-D } D^{\text {mom }}\end{array}$ & & $\begin{array}{l}82(17) \\
78(9)\end{array}$ & $\begin{array}{r}92(24) \\
100(11)\end{array}$ & $\begin{array}{l}97(34) \\
88(25)\end{array}$ & $\begin{array}{l}82(39) \\
89(38)\end{array}$ & $\begin{array}{l}65(17) \\
79(24)\end{array}$ & \\
\hline$f s(1) K 10$ mRNA & $\begin{array}{l}\text { wild-type } \\
\text { Bic-D } D^{\text {mom }}\end{array}$ & $\begin{array}{r}100(16) \\
95(20)\end{array}$ & $\begin{array}{l}100(22) \\
100(21)\end{array}$ & $\begin{array}{l}90(31) \\
87(23)\end{array}$ & $\begin{array}{l}88(33) \\
70(20)\end{array}$ & $\begin{array}{l}67(39) \\
35(23)\end{array}$ & $\begin{array}{r}69(16) \\
\mathbf{0}(14)\end{array}$ & $\begin{array}{r}55(38) \\
\mathbf{0}(21)\end{array}$ \\
\hline oskar mRNA & $\begin{array}{l}\text { wild-type } \\
\text { Bic-D } D^{\text {mom }}\end{array}$ & $\begin{array}{r}100(47) \\
69(26)\end{array}$ & $\begin{array}{l}92(26) \\
66(6)\end{array}$ & $\begin{array}{l}89(28) \\
67(21)\end{array}$ & $\begin{array}{l}\mathbf{8 3}(29) \\
\mathbf{5 2}(23)\end{array}$ & $\begin{array}{l}75(44) \\
48(27)\end{array}$ & $\begin{array}{l}81(36) \\
32(19)\end{array}$ & $\begin{array}{l}63(48) \\
21(24)\end{array}$ \\
\hline kinesin- $\beta$-gal* & $\begin{array}{l}\text { wild-type } \\
\text { Bic- } D^{\text {mom }}\end{array}$ & & & $\begin{array}{l}\mathbf{5 5}(11) \\
\mathbf{4 0}(5)\end{array}$ & $\begin{array}{l}87(55) \\
95(40)\end{array}$ & $\begin{array}{r}100(30) \\
93(14)\end{array}$ & $\begin{array}{r}84(31) \\
100(14)\end{array}$ & $\begin{array}{r}\mathbf{0}(15) \\
\mathbf{1 0}(21)\end{array}$ \\
\hline
\end{tabular}

Numbers in bold represent the percentage of egg chambers in which the localized factor is detected at its usual site of accumulation: grk RNA, anterior oocyte in stage 7, perinuclear in stages 8-10A; $f_{s}(1) K 10$ (and orb), oocyte in stages 2-6, anterior oocyte in stages 7-10B; osk, oocyte in stages 2-6, anterior in stages 7-8, posterior in stages 8 -10B; kinesin- $\beta$-gal, posterior in stages 8 -10A.

Numbers in parentheses represent total number of egg chambers examined for each stage.

*Localization of the kinesin- $\beta$-gal fusion protein as visualized by $\beta$-gal staining. 

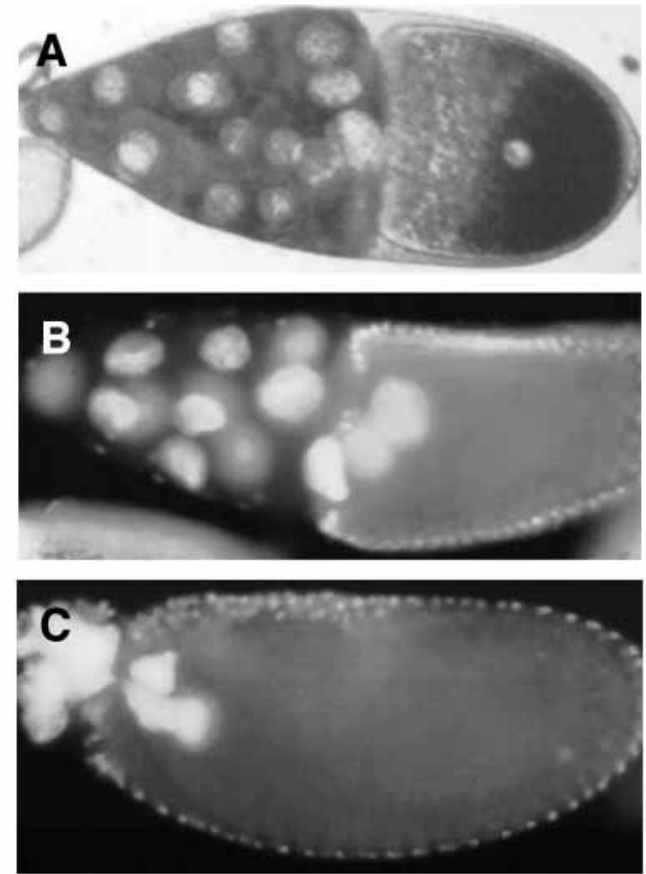

Fig. 4. (A) Mislocalization of the oocyte nucleus in a stage 10B Bic$D^{\text {mom }}$ egg chamber. (B) Hoechst staining of a stage 10B Bic- $D^{\text {mom }}$ egg chamber revealing centripetal cell migration between nurse cells. (C) A Hoechst stained stage 12 Bic- $D^{m o m}$ egg chamber in which nurse cell nuclei have become trapped within the maturing egg.

oocyte. In either case, these results do not explain the oocyte growth defect in Bic- $D^{m o m}$ egg chambers, and we predict that other germ line components fail to localize to the oocyte in these mutants.

\section{$B i c-D$ requirement in localizing patterning factors within the oocyte}

In wild-type oogenesis, osk mRNA appears transiently at the anterior of the oocyte in stage 8, and then starts to accumulate at the posterior where it stays for the remainder of oogenesis. In ovaries from females carrying Bic-D dominant mutations, some osk mRNA is retained at the anterior of the oocyte late in oogenesis, while the posterior accumulation of osk is not noticeably affected (Ephrussi et al., 1991; Kim-Ha et al., 1991). To determine if loss of Bic-D activity in later oogenesis also affects osk mRNA localization, we examined the distribution of osk transcripts in stage 8 to $10 \mathrm{~B} \mathrm{Bic-D^{mom }}$ egg chambers. The transient anterior accumulation and early posterior accumulation of $o s k$ transcript is normal, but in stages 9 and 10, the posterior accumulation becomes progressively reduced and often no localized transcript is detected (Table 2; Fig. 5B). This effect on osk is clearly distinct from that exerted by dominant Bic-D mutations, indicating that the Bic-D dominant alleles are neomorphs with respect to their effect on osk localization. Because eggs with a high expressivity of the Bic- $D^{m o m}$ phenotype are not fertilized, we have not been able to determine if the failure to maintain osk localization in mutant egg chambers results in production of embryos with abdominal defects.

To determine whether Bic-D is required specifically for the localization of osk mRNA or more generally for RNA localization within the oocyte, we examined the distribution of two other localized transcripts, orb and $f s(1) K 10$ in late stage Bic$D^{m o m}$ egg chambers. These mRNAs normally accumulate at the anterior margin in stage 8 where they remain until late in stage 10 (Lantz et al., 1992; Cheung et al., 1992). In Bic-D ${ }^{\text {mom }}$ egg chambers, both of these transcripts initially accumulate at the anterior in stage 8 but, in later stages, the amount of localized transcript is reduced or undetectable (Table 2, Fig. $w t$
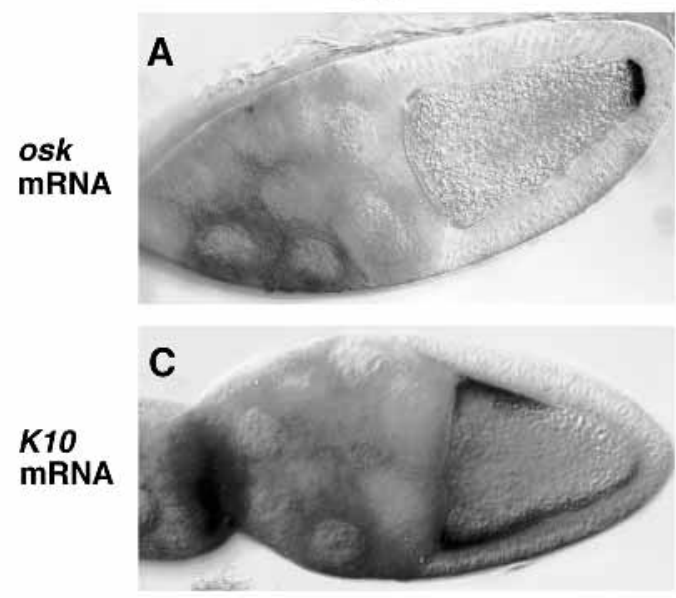

kin-lacZ
$B i c-D^{m o m}$
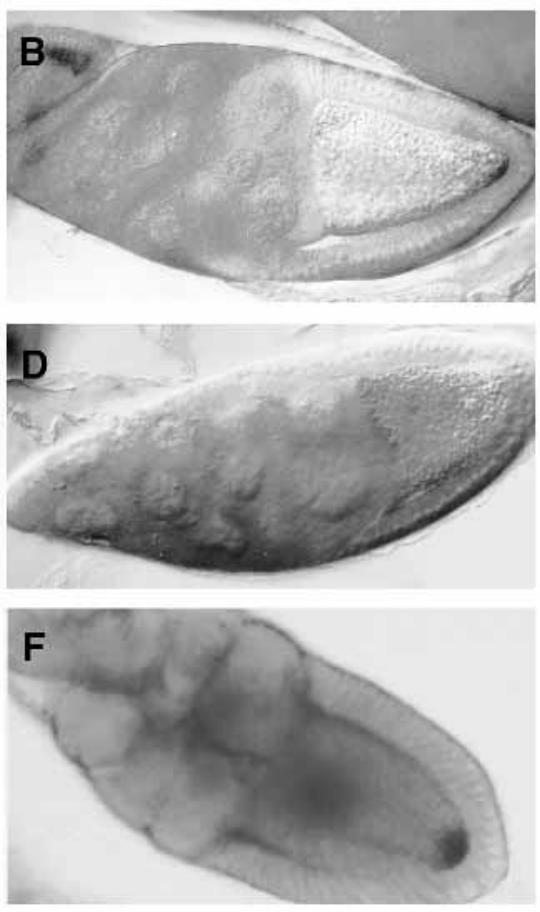

Fig. 5. Distribution of localized factors in Bic- $D^{\text {mom }}$ egg chambers.

(A) Posterior accumulation of $o s k$ transcripts in a stage 10 control egg chamber. (B) Low level posterior accumulation of $o s k$ transcripts in a stage 10 Bic- $D^{\text {mom }}$ egg chamber.

(C) $f s(1) K 10$ transcript accumulation at the anterior margin of the oocyte in a late stage 9 control egg chamber. (D) Absence of $f_{s}(1) K 10$ transcripts at the anterior in a late stage $9 \mathrm{Bic}-\mathrm{D}^{\mathrm{mom}}$ egg chamber. The same results were seen with orb transcripts (not shown). (E,F) $\beta$-galactosidase activity from the kinesin-lac $Z$ fusion gene is localized to the posterior pole in stage 10A of oogenesis in control and Bic- $D^{\text {mom }}$ egg chambers. 
5D). Therefore, Bic-D seems to be required for the proper transport or maintenance of anteriorly and posteriorly localized mRNAs in the oocyte late in oogenesis. It is formally possible that the failure to localize factors late in oogenesis reflects a continued requirement for their transport into the oocyte, and that it is this process that is defective in Bic- $D^{m o m}$. This is, however, unlikely since Bic-D does not appear to be required for nurse-cell-to-oocyte transport late in oogenesis (Table 1).

One mechanism by which Bic-D may be involved in the localization of anteriorly and posteriorly localized mRNAs is through the organization of microtubules in the oocyte. To test this hypothesis, we looked at the localization of a kinesin$\beta$-gal fusion protein in Bic- $D^{\text {mom }}$ egg chambers. This fusion protein normally accumulates at the posterior pole in stages 8 to $10 \mathrm{~A}$ dependent on its activity as a plus-end-directed microtubule motor (Clark et al., 1994) and dependent on proper microtubule organization in the oocyte (Clark et al., 1994; Lane and Kalderon, 1994; González-Reyes and St. Johnston, 1994). In Bic- $D^{\text {mom }}$ egg chambers, during the stages when we observe a reduction in osk mRNA at the posterior, kinesin- $\beta$-gal is still localized normally at this site (Fig. 5F; Table 2). Therefore, Bic-D does not seem to be required during late oogenesis for microtubule organization within the oocyte.

\section{DISCUSSION}

\section{Bic-D role in dorsal/ventral patterning}

In wild-type oogenesis, localized grk signal is thought to activate the top/EGF receptor in overlying columnar follicle cells, setting off a signal transduction cascade that leads to the establishment of dorsal/anterior follicle cell fate. This newly established fate is first seen in the expression of specific marker genes. Later in oogenesis, these cells secrete specialized dorsal/anterior chorionic structures, the dorsal appendages (Schüpbach and Roth, 1994).

In Bic- $D^{\text {mom }}$ egg chambers, the posterior displacement of the oocyte and grk signal correlates with a posterior shift in expression of the dorsal/anterior columnar follicle cell marker BB142, and this also correlates with a posterior displacement of the dorsal appendages. However, the posteriorly displaced appendages are typically reduced and often no dorsal appendage material is formed at all (Fig. 2C,E). This is surprising given that BB142 expression, though displaced, reaches levels indistinguishable from those in wild type (Fig. $3 \mathrm{~F}$ ). While it may be that grk signaling to posterior follicle cells is in fact slightly reduced, it is also possible that the failure to properly make dorsal appendages is due to an inability of posterior follicle cells to adopt this fate in response to grk signaling. This latter possibility is supported by experiments in which an activated form of $D$-Raf is expressed in all follicle cells (Brand and Perrimon, 1994). Induction of this transgene results in the expression of the dorsal/anterior follicle cell marker AN296 in all follicle cells overlying the oocyte, but only those follicle cells in a ring at the anterior respond by secreting dorsal appendage material. These results, along with our own findings can be explained if anterior columnar follicle cells are fully competent to make dorsal appendages in response to grk signaling whereas more posterior follicle cells are not. This may imply that the columnar follicle cells are prepatterned along their anterior/posterior axis prior to reception of grk signal.

\section{Autonomous patterning of follicle cell fates}

Normally by stage 10 of oogenesis, follicle cell identities correspond tightly with the position of these cells relative to the underlying germ line. The anteriormost flattened follicle cells overly the nurse cells. The remaining follicle cells make up a columnar epithelium, which precisely covers the oocyte. The most anterior of these columnar follicle cells overly the nurse cell/oocyte boundary and later initiate centripetal migration at this site. Also, as described above, only the follicle cells overlying the anterior of the oocyte appear to be competent to respond properly to the anteriorly localized grk signal.

In Bic- $D^{m o m}$, even though the oocyte is smaller than in wild type, the position of the different follicle cell types is unaffected. Approximately the same number of follicle cells become columnar and migrate posteriorly. As a result, by stage $10 \mathrm{~A}$ the most anteriorly situated of these come to overly nurse cells instead of the oocyte (Figs 3D, 4B). Therefore, follicle cell thickening and posterior migration are not dependent on the size of the oocyte. Centripetal cells are also determined independent of their position relative to the germ line. In Bic$D^{\text {mom }}$ egg chambers, these anterior columnar follicle cells still express the centripetal cell marker BB127, and usually initiate centripetal cell migration even though they do not overly the nurse cell/oocyte boundary (Fig. 4B,C). Therefore, there is evidence for a prepatterning of follicle cell fates along the $\mathrm{A} / \mathrm{P}$ axis independent of their position relative to the underlying germ line.

\section{Bic- $D$ role in localizing factors within the egg chamber}

Indirect evidence has pointed to a role for microtubule-based transport or anchoring in the transfer of nurse cell contents into the oocyte. Beginning in region $2 b$ of the germarium, around the time that specific factors begin to accumulate in the oocyte, microtubules are arranged with minus ends in the oocyte and plus ends extending through ring canals into the other 15 nurse cells (Theurkauf et al., 1992). In egg chambers treated with microtubule-destabilizing drugs, the presumptive oocyte adopts a nurse cell fate (Koch and Spitzer, 1983) and oocytespecific factors fail to accumulate in a single cell (Theurkauf et al., 1993). Recessive alleles of Bic-D result in a similar block in oocyte differentiation. In these mutant ovaries, failure to make an oocyte correlates with a failure to localize oocytespecific mRNAs and proteins, and failure to form a visible microtubule organizing center in the presumptive oocyte (Suter and Steward, 1991; Theurkauf et al., 1993; Ran et al., 1994).

Bic- $D$ could therefore be either a factor required in the prooocyte for differentiation of an oocyte, or a component of a transport or anchoring system that localizes such factors to the presumptive oocyte. Studying the precise role of Bic-D in oocyte determination is hampered by the small size of the germ line cells in these early stages and by the fact that lack of Bic$D$ causes the presumptive oocyte to adopt a nurse cell fate, inducing many cellular changes that do not directly depend on $B i c-D$. Analyzing the later requirements for $B i c-D$ in oogenesis circumvents these problems and should thus allow us to test hypotheses for molecular roles of Bic-D.

From this analysis, we have found that $B i c-D$ is required for 
oocyte growth in early oogenesis, corresponding to the stages when microtubules are asymmetrically arranged within the 16cell cyst such that they could support directed transport into the oocyte. Treatment of wild-type egg chambers with microtubule-destabilizing drugs during these stages also leads to reduced oocyte growth (Koch and Spitzer, 1983), suggesting that both Bic-D and microtubules are involved in nurse-cellto-oocyte transport early in oogenesis. These findings support the idea that the oocyte determination block in Bic-D mutants and in flies treated with microtubule-disrupting drugs is due primarily to a block in transport into the presumptive oocyte.

We have also found that Bic-D is required for the localization or maintenance of $f_{S}(1) K 10$ and orb transcripts at the anterior of the oocyte and osk mRNA at the posterior of the oocyte late in oogenesis (stages 9 to 10B, Fig. 5B,D, Table 2). Inhibitor studies have shown that transport and/or maintenance of these localized transcripts depends on microtubules (Pokrywka and Stephenson, 1991; Clark et al., 1994; Pokrywka and Stephenson, 1995). Bic-D is also required late in oogenesis to maintain the correct positioning of the oocyte nucleus (Fig. 4A), a process that is also dependent on microtubules (Koch and Spitzer, 1983). Taken together, these results suggest that Bic-D may be part of a microtubule-based transport or anchoring system that is utilized repeatedly throughout oogenesis.

One possible role for Bic-D in oocyte determination is in the establishment of the polarized microtubule network that connects the presumptive oocyte to the other 15 germ-line cells. If this is the case, the later requirements for Bic-D might reflect a similar role in organizing microtubules later in oogenesis. Three different results argue that this is not the case. First, using the kinesin- $\beta$-gal fusion protein as a marker for microtubule polarity, we find that, in Bic- $D^{\text {mom }}$, microtubules are organized correctly in stages 8 to $10 \mathrm{~B}$ of oogenesis (Fig. $5 \mathrm{~F})$. Second, the microtubule-dependent rapid ooplasmic streaming, which begins in stage 10B, is not affected. Third, immunostaining of mutant egg chambers with an anti-tubulin antibody reveals no effects on microtubule organization throughout oogenesis (data not shown).

Our results favor a model in which Bic-D is involved in the transport or anchoring of localized factors along an existing microtubule network early and late in oogenesis. Bic-D does not seem to encode a microtubule-based motor (Suter et al., 1989; Wharton and Struhl, 1989), but it may act as an intermediate in either transport or anchoring of factors to microtubules. Such intermediate proteins have been described in other systems: the dynactin complex appears to mediate interactions between membrane-bound organelles and the microtubule-based motor dynein (Schroer and Sheetz, 1991). Similarly, CLIP 170 appears to function in the anchoring of endocytic carrier vesicles to microtubules (Pierre et al., 1992). By analogy, similar proteins would be expected to mediate the interactions between localized factors and microtubules in the Drosophila egg chamber. It is not yet possible to distinguish between a transport or anchoring role for Bic-D. One way to distinguish between these models will be to identify the factors that interact with Bic-D and to follow their movement in wild type and in Bic- $D^{\text {mom }}$ egg chambers.

We wish to thank Thuy Nguyen, Akira Nakamura and Stéphane Larochelle for critical reading of the manuscript. Thanks to Trudi
Schüpbach for providing us with gurken clones and alleles and enhancer trap lines. Thanks to Paul Schedl for the pElba plasmid and to Ira Clark for the kinesin-lacZ stock. This research is supported by the National Sciences and Engineering Research Council of Canada and by the National Cancer Institute of Canada with funds from the Canadian Cancer Society and the Terry Fox Run. B. S. is a Research Scientist of the National Cancer Institute of Canada supported by funds from the Canadian Cancer Society.

\section{REFERENCES}

Bohrmann, J., Frey, A. and Gutzeit, H. O. (1992). Observations on the polarity of mutant Drosophila follicles lacking the oocyte. Roux Arch. Dev. Biol. 201, 268-274.

Bohrmann, J., and Biber, K. (1994). Cytoskeleton-dependent transport of cytoplasmic particles in previtellogenic to mid-vitellogenic ovarian follicles of Drosophila: Time-lapse analysis using video-enhanced contrast microscopy. J. Cell Sci. 107, 849-858

Brand, A. H. and Perrimon, N. (1994). Raf acts downstream of the EGF receptor to determine dorsoventral polarity during Drosophila oogenesis. Genes Dev. 8, 629-639.

Cheung, H.-K., Serano, T. L. and Cohen, R. S. (1992). Evidence for a highly selective RNA transport system and its role in establishing the dorsoventral axis of the Drosophila egg. Development 114, 653-661.

Clark, I., Giniger, E., Ruohola-Baker, H., Jan, L. Y. and Jan, Y. N. (1994) Transient posterior localization of a kinesin fusion protein reflects anteroposterior polarity of the Drosophila oocyte. Current Biol. 4, 289-300.

Cooley, L., and Theurkauf, W.E. (1994). Cytoskeletal functions during Drosophila oogenesis. Science 266, 590-596.

Ephrussi, A., Dickinson, L. K. and Lehmann, R. (1991). osk organizes the germ plasm and directs localization of the posterior determinant nanos. Cell 66, 37-50.

González-Reyes, A. and St. Johnston, R. D. (1994). Role of oocyte position in establishment of anterior-posterior polarity in Drosophila. Science 266, 639642

Kellum, R. and Schedl, P. (1991). A positon-effect assay for boundaries of higher order chromosomal domains. Cell 64, 941-950.

King, R. C. and Burnett, R. G. (1959). An autoradiographic study of uptake of tritiated glycine, thymidine, and uridine by fruit fly ovaries. Science $\mathbf{1 2 9}$ 1674-1675.

Kim-Ha, J., Smith, J. L. and Macdonald, P. M. (1991). osk mRNA is localized to the posterior pole of the Drosophila oocyte. Cell 66, 23-35.

Koch, E. A. and Spitzer, R. H. (1983). Multiple effects of colchicine on oogenesis in Drosophila: Induced sterility and switch of potential oocyte to nurse-cell developmental pathway. Cell Tissue Res. 228, 21-32.

Lane, M. E. and Kalderon, D. (1994). RNA localization along the anteroposterior axis of the Drosophila oocyte requires PKA-mediated signal transduction to direct normal microtubule organization. Genes Dev. 8, 2986 2995.

Lantz, V., Ambrosio, L. and Schedl, P. (1992). The Drosophila orb gene is predicted to encode sex-specific germline RNA-binding proteins and has localized transcripts in ovaries and early embryos. Development 115, 75-88.

Lis, J. T., Simon, J. A. and Sutton, C. A. (1983). New heat shock puffs and $\beta$ galactosidase activity resulting from transformation of Drosophila with an hsp70-lacZ hybrid gene. Cell 35, 403-410.

Mohler, J. and Wieschaus, E. F. (1986). Dominant maternal-effect mutations of Drosophila melanogaster causing the production of double-abdomen embryos. Genetics 112, 803-822.

Neuman-Silberberg, F. S. and Schüpbach, T. (1993). The Drosophila dorsoventral patterning gene gurken produces a dorsally localized RNA and encodes a TGFa-like protein. Cell 75, 165-174.

Pierre, P., Scheel, J., Rickard, J. E. and Kreis, T. E. (1992). CLIP-170 links endocytic vesicles to microtubules. Cell 70, 887-900.

Pokrywka, N. J. and Stephenson, E. C. (1991). Microtubules mediate the localization of bicoid RNA during Drosophila oogenesis. Development 113, 55-66.

Pokrywka, N. J. and Stephenson, E. C. (1995). Microtubules are a general component of mRNA localization systems in Drosophila oocytes. Dev. Biol. 167, 363-370.

Ran, B., Bopp, R. and Suter, B. (1994). Null alleles reveal novel requirements for Bic-D during Drosophila oogenesis and zygotic development. Development 120, 1233-1242. 


\section{A. Swan and B. Suter}

Roth, S. and Schüpbach, T. (1994). The relationship between ovarian and embryonic dorsoventral patterning in Drosophila. Development 120, 2245 2257.

Schroer, T. A. and Sheetz, M. P. (1991). Two activators of microtubule-based vesicle transport. J. Cell Biol. 115, 1390-1318.

Schüpbach, T. (1987). Germ line and soma cooperate during oogenesis to establish the dorsoventral pattern of egg shell and embryo in Drosophila melanogaster. Cell 49, 699-707.

Schüpbach, T. and Wieschaus, E. (1991). Female sterile mutations on the second chromosome of Drosophila melanogaster: II Mutations blocking oogenesis or altering egg morphology. Genetics 129, 1119-1136.

Schüpbach, T. and Roth, S. (1994). Dorsoventral patterning in Drosophila oogenesis. Curr. Opin. Genet. Dev. 4, 502-507.

Suter, B., Romberg, L. M. and Steward, R. (1989). Bicaudal-D, a Drosophila gene involved in developmental asymmetry: localized transcript accumulation in ovaries and sequence similarity to myosin heavy chain tail domains. Genes Dev. 3, 1957-1968.

Suter, B. and Steward, R. (1991). Requirement for phosphorylation and localization of the Bicaudal-D protein in Drosophila oocyte differentiation. Cell 67, 917-926.

Theurkauf, W. E., Smiley, S., Wong, M. L. and Alberts, B. M. (1992) Reorganization of the cytoskeleton during Drosophila oogenesis: implications for axis specification and intercellular transport. Development 115, 923-936.

Theurkauf, W. E., Alberts, B. M., Jan, Y. N. and Jongens, T. A. (1993). A central role for microtubules in the differentiation of Drosophila oocytes. Development 118, 1169-1180.

Wharton, R. P. and Struhl, G. (1989). Structure of the Drosophila Bicaudal$\mathrm{D}$ protein and its role in localizing the posterior determinant nanos. Cell 59, 881-892.

Wieschaus, E. and Nüsslein-Volhard, C. (1986). Looking at embryos. In Drosophila: a Practical Approach (ed. D. B. Roberts), pp. 199-227. Oxford: IRL Press. 